\title{
A PARALLEL HYBRID METHOD FOR EQUILIBRIUM PROBLEMS, VARIATIONAL INEQUALITIES AND NONEXPANSIVE MAPPINGS IN HILBERT SPACE
}

\author{
DANG VAN Hieu
}

\begin{abstract}
In this paper, a novel parallel hybrid iterative method is proposed for finding a common element of the set of solutions of a system of equilibrium problems, the set of solutions of variational inequalities for inverse strongly monotone mappings and the set of fixed points of a finite family of nonexpansive mappings in Hilbert space. Strong convergence theorem is proved for the sequence generated by the scheme. Finally, a parallel iterative algorithm for two finite families of variational inequalities and nonexpansive mappings is established.
\end{abstract}

\section{Introduction}

Let $H$ be a real Hilbert space with the inner product $\langle\cdot, \cdot\rangle$ and the norm $\|\cdot\|$. Let $C$ be a nonempty closed convex subset of $H$. Let $A: C \rightarrow H$ be a (nonlinear) operator. The variational inequality problem is to find $p^{*} \in C$ such that

$$
\left\langle A p^{*}, p-p^{*}\right\rangle \geq 0, \quad \forall p \in C .
$$

The set of solutions of (1) is denoted by $V I(A, C)$.

A mapping $S: C \rightarrow C$ is said to be nonexpansive if $\|S x-S y\| \leq\|x-y\|$ for all $x, y \in C$. The set of fixed points of $S$ is denoted by

$$
F(S)=\{x \in C: S(x)=x\} .
$$

For finding a common element of the set of fixed points of a nonexpansive mapping and the set of solutions of the variational inequality for an $\alpha$-inverse strongly monotone mapping in Hilbert space, Takahashi and Toyoda [17] proposed the following iterative method: $x_{0} \in C$ and

$$
x_{n+1}=\alpha_{n} x_{n}+\left(1-\alpha_{n}\right) S P_{C}\left(x_{n}-\lambda_{n} A x_{n}\right)
$$

Received May 18, 2014; Revised December 18, 2014.

2010 Mathematics Subject Classification. 65Y05, 47H09, 47H10, 47J20.

Key words and phrases. hybrid method, equilibrium problem, variational inequality, parallel computation. 
for $n=0,1,2, \ldots$, where $\lambda_{n} \in[a, b]$ for some $a, b \in(0,2 \alpha)$ and $\alpha_{n} \in[c, d]$ for some $c, d \in(0,1)$. They proved that the sequence $\left\{x_{n}\right\}$ converges weakly to $z \in F(S) \cap V I(A, C)$, where $z=\lim _{n \rightarrow \infty} P_{F(S) \cap V I(A, C)} x_{n}$. To obtain strong convergence, Iiduka and Takahashi [11] proved the following convergence theorem:

Theorem 1.1 ([11]). Let $C$ be a closed convex subset of a real Hilbert space $H$. Let $A$ be an $\alpha$-inverse-strongly-monotone mapping of $C$ into $H$ and let $S$ be a nonexpansive nonself-mapping of $C$ into $H$ such that $F(S) \cap V I(A, C) \neq \emptyset$. Suppose $x_{1}=x \in C$ and $\left\{x_{n}\right\}$ is given by

$$
x_{n+1}=P_{C}\left(\alpha_{n} x_{n}+\left(1-\alpha_{n}\right) S P_{C}\left(x_{n}-\lambda_{n} A x_{n}\right)\right)
$$

for every $n=1,2, \ldots$, where $\left\{\alpha_{n}\right\}$ is a sequence in $[0,1)$ and $\left\{\lambda_{n}\right\}$ is a sequence in $[0,2 \alpha]$. If $\left\{\alpha_{n}\right\}$ and $\left\{\lambda_{n}\right\}$ are chosen so that $\lambda_{n} \in[a, b]$ for some $a, b$ with $0<a<b<2 \alpha$,

$$
\lim _{n \rightarrow \infty} \alpha_{n}=0, \quad \sum_{n=1}^{\infty} \alpha_{n}=\infty, \quad \sum_{n=1}^{\infty}\left|\alpha_{n+1}-\alpha_{n}\right|<\infty, \quad \sum_{n=1}^{\infty}\left|\lambda_{n+1}-\lambda_{n}\right|<\infty,
$$

then $\left\{x_{n}\right\}$ converges strongly to $P_{F(S) \cap V I(A, C)} x$.

Let $f$ be a bifunction from $C \times C$ to the set of real numbers $\mathbb{R}$. The equilibrium problem for $f$ is to find an element $\widehat{x} \in C$, such that

$$
f(\widehat{x}, y) \geq 0, \forall y \in C \text {. }
$$

The set of solutions of the equilibrium problem (2) is denoted by $\operatorname{EP}(f)$. Equilibrium problems are generalized by several problems such as: optimization problems, variational inequalities, etc. In recent years, several methods have been proposed for finding a solution of equilibrium problem (2) in Hilbert space $[5,7,16,18,19]$.

In 2010, for finding a common element of the set of fixed points of nonexpansive mappings, the set of the solutions of variational inequalities for $\alpha$ inverse strongly monotone operators, and the set of the solutions of equilibrium problems in Hilbert space, Saeidi [12] proposed the following iterative method: $x_{0} \in H$ and

$$
\left\{\begin{array}{l}
u_{n}=T_{r_{M, n}}^{f_{M}} \cdots T_{r_{1, n}}^{f_{1}} x_{n}, \\
v_{n}=P_{C}\left(I-\lambda_{N, n} A_{N}\right) \cdots P_{C}\left(I-\lambda_{1, n} A_{1}\right) u_{n}, \\
y_{n}=\left(1-\alpha_{n}\right) x_{n}+\alpha_{n} W_{n} v_{n}, \\
C_{n}=\left\{v \in H:\left\|v-y_{n}\right\| \leq\left\|v-x_{n}\right\|\right\}, \\
Q_{n}=\left\{v \in H:\left\langle x_{0}-x_{n}, x_{n}-v\right\rangle \geq 0\right\}, \\
x_{n+1}=P_{C_{n} \cap Q_{n}} x_{0}, n \geq 1,
\end{array}\right.
$$

where $W_{n}$ is the nonexpansive mapping, so-called the $W$-mapping [14], and $T_{r}^{f} x:=u$ is the unique solution to the regularized equilibrium problem

$$
f(u, y)+\frac{1}{r}\langle y-u, u-x\rangle \geq 0, \quad \forall y \in C .
$$


Clearly, Saeidi's algorithm is inherently sequential. Hence, when the numbers of operators $N$ and bifunctions $M$ are large, it is costly on a single processor.

Very recently, Anh and Chung [2] have proposed the following parallel hybrid iterative method for finding an element of the set of fixed points of a finite family of relatively nonexpansive mappings $\left\{S_{i}\right\}_{i=1}^{N}$ :

$$
\left\{\begin{array}{l}
x_{0} \in C_{0}:=C, Q_{0}:=C, \\
y_{n}^{i}=\alpha_{n} x_{n}+\left(1-\alpha_{n}\right) S_{i} x_{n}, i=1, \ldots, N, \\
i_{n}:=\arg \max \left\{\left\|y_{n}^{i}-x_{n}\right\|: i=1, \ldots, N\right\}, \bar{y}_{n}:=y_{n}^{i_{n}}, \\
C_{n}=\left\{v \in C:\left\|v-\bar{y}_{n}\right\| \leq\left\|v-x_{n}\right\|\right\} \\
Q_{n}=\left\{v \in C:\left\langle x_{0}-x_{n}, x_{n}-v\right\rangle \geq 0\right\} \\
x_{n+1}=P_{C_{n} \cap Q_{n}} x_{0}, n \geq 0 .
\end{array}\right.
$$

This algorithm was extended by Anh and Hieu [3] for a finite family of asymptotically quasi $\phi$-nonexpansive mappings in Banach spaces.

In this paper, motivated by the results of Takahashi et al. [11, 17], Saeidi [12], Anh and Chung [2], we propose the following novel parallel hybrid iterative method for finding a common element of the set of solutions of a system of equilibrium problems for bifunctions $\left\{f_{l}\right\}_{l=1}^{K}$, the set of solutions of variational inequalities for $\alpha$-inverse strongly monotone mappings $\left\{A_{k}\right\}_{k=1}^{M}$ and the set of fixed points of a finite family of nonexpansive mappings $\left\{S_{i}\right\}_{i=1}^{N}$ :

$$
\left\{\begin{array}{l}
x_{0} \in H, C_{0}=Q_{0}=C, \\
z_{n}^{l}=T_{r_{n}}^{f_{l}} x_{n}, l=1, \ldots, K, \\
l_{n}:=\arg \max \left\{\left\|z_{n}^{l}-x_{n}\right\|: l=1, \ldots, K\right\}, \bar{z}_{n}:=z_{n}^{l_{n}}, \\
u_{n}^{k}=P_{C}\left(\bar{z}_{n}-\lambda A_{k} \bar{z}_{n}\right), k=1, \ldots, M, \\
k_{n}:=\arg \max \left\{\left\|u_{n}^{k}-x_{n}\right\|: k=1, \ldots, M\right\}, \bar{u}_{n}:=u_{n}^{k_{n}}, \\
y_{n}^{i}=\alpha_{n} \bar{u}_{n}+\left(1-\alpha_{n}\right) S_{i} \bar{u}_{n}, i=1, \ldots, N, \\
i_{n}:=\arg \max \left\{\left\|y_{n}^{i}-x_{n}\right\|: i=1, \ldots, N\right\}, \bar{y}_{n}:=y_{n}^{i_{n}}, \\
C_{n}=\left\{v \in H:\left\|v-\bar{y}_{n}\right\| \leq\left\|v-\bar{z}_{n}\right\| \leq\left\|v-x_{n}\right\|\right\}, \\
Q_{n}=\left\{v \in H:\left\langle x_{0}-x_{n}, x_{n}-v\right\rangle \geq 0\right\}, \\
x_{n+1}=P_{C_{n} \cap Q_{n}} x_{0}, n \geq 0,
\end{array}\right.
$$

where $\lambda \in(0,2 \alpha)$ and the control parameter sequences $\left\{\alpha_{n}\right\},\left\{r_{n}\right\}$ satisfy some conditions. Clearly, in the method (3), at $n^{\text {th }}$ step, we can calculate the intermediate approximations $z_{n}^{l}$ in parallel. Then, among all $z_{n}^{l}$, the element $\bar{z}_{n}$ which is farest from $x_{n}$ is selected. Using the element $\bar{z}_{n}$ to find the approximations $u_{n}^{k}$ in parallel. After that, we chose the element $\bar{u}_{n}$ that is farest from $x_{n}$ among $u_{n}^{k}$. Similarly, $y_{n}^{i}$ are calculated in parallel and $\bar{y}_{n}$ is determined. Based on $\bar{y}_{n}, \bar{z}_{n}, x_{n}$, the closed and convex subsets $C_{n}, Q_{n}$ are constructed. Finally, the next approximation $x_{n+1}$ is determined as the projection of $x_{0}$ onto the intersection $C_{n} \cap Q_{n}$ of two closed and convex subsets $C_{n}$ and $Q_{n}$. 
This paper is organized as follows: In Section 2, we collect some definitions and results for researching into the convergence of the proposed method. Section 3 deals with the convergence analysis of the method and its applications.

\section{Preliminaries}

In what follows, we review some definitions and results, which are employed in this paper. We refer the reader to [11]. We write $x_{n} \rightarrow x$ to indicate that the sequence $\left\{x_{n}\right\}$ converges strongly to $x$ and $x \rightarrow x$ implies that $\left\{x_{n}\right\}$ converges weakly to $x$.

A mapping $A: C \rightarrow H$ is called $\alpha$-inverse strongly monotone if there exists a constant $\alpha>0$ such that

$$
\langle A x-A y, x-y\rangle \geq \alpha\|A x-A y\|^{2}
$$

for all $x, y \in C$ and $\eta$-strongly monotone if there exists $\eta>0$ such that

$$
\langle A x-A y, x-y\rangle \geq \eta\|x-y\|^{2} .
$$

It is well known that if $A$ is $\eta$-strongly monotone and $L$-Lipschitz, i.e.,

$$
\|A x-A y\| \leq L\|x-y\|
$$

for all $x, y \in C$, then $A$ is $\eta / L^{2}$-inverse strongly monotone. If $A: C \rightarrow H$ is $\alpha$-inverse strongly monotone, then $A$ is $1 / \alpha$-Lipschitz continuous and $I-\lambda A$ is nonexpansive of $C$ onto $H$, where $\lambda \in(0,2 \alpha)$. If $T$ is nonexpansive, then $A=I-T$ is $1 / 2$-inverse strongly monotone and $V I(A, C)=F(T)$.

For every $x \in H$, the element $P_{C} x$ is defined by

$$
P_{C} x=\arg \min \{\|y-x\|: y \in C\} .
$$

Since $C$ is a nonempty closed and convex subset of $H, P_{C} x$ is existent and unique. Mapping $P_{C}: H \rightarrow C$ is called the projection of $H$ onto $C$. It is also known that $P_{C}$ satisfies

$$
\left\langle P_{C} x-P_{C} y, x-y\right\rangle \geq\left\|P_{C} x-P_{C} y\right\|^{2} .
$$

This implies that $P_{C}$ is 1-inverse strongly monotone and for all $x \in C, y \in H$, we have

$$
\left\|x-P_{C} y\right\|^{2}+\left\|P_{C} y-y\right\|^{2} \leq\|x-y\|^{2} .
$$

Moreover, $z=P_{C} x$ if only if

$$
\langle x-z, z-y\rangle \geq 0, \quad \forall y \in C,
$$

and this implies that $p^{*} \in V I(A, C)$ if only if

$$
p^{*}=P_{C}\left(p^{*}-\lambda A p^{*}\right), \quad \lambda>0 .
$$

We have the following result of the convexity and closedness of $V I(A, C)$.

Lemma 2.1 ([15]). Let $C$ be a nonempty, closed convex subset of a Banach space $E$ and $A$ be a monotone, hemicontinuous operator of $C$ into $E^{*}$. Then

$$
V I(A, C)=\{u \in C:\langle v-u, A v\rangle \geq 0 \text { for all } v \in C\} \text {. }
$$


Next, for solving the equilibrium problem (2), we assume that the bifunction $f$ satisfies the following conditions:

(A1) $f(x, x)=0$ for all $x \in C$;

(A2) $f$ is monotone, i.e., $f(x, y)+f(y, x) \leq 0$ for all $x, y \in C$;

(A3) For all $x, y, z \in C$,

$$
\lim _{t \rightarrow 0^{+}} \sup f(t z+(1-t) x, y) \leq f(x, y) ;
$$

(A4) For all $x \in C, f(x, \cdot)$ is convex and lower semicontinuous.

The following results concern with the bifunction $f$ :

Lemma $2.2([7])$. Let $C$ be a closed and convex subset of Hilbert space $H, f$ be a bifunction from $C \times C$ to $\mathbb{R}$ satisfying the conditions (A1)-(A4) and let $r>0, x \in H$. Then, there exists $z \in C$ such that

$$
f(z, y)+\frac{1}{r}\langle y-z, z-x\rangle \geq 0, \quad \forall y \in C .
$$

Lemma $2.3([7])$. Let $C$ be a closed and convex subset of a Hilbert space $H$, $f$ be a bifunction from $C \times C$ to $\mathbb{R}$ satisfying the conditions (A1)-(A4). For all $r>0$ and $x \in H$, define the mapping

$$
T_{r}^{f} x=\left\{z \in C: f(z, y)+\frac{1}{r}\langle y-z, z-x\rangle \geq 0, \quad \forall y \in C\right\} .
$$

Then the following hold:

(B1) $T_{r}^{f}$ is single-valued;

(B2) $T_{r}^{f}$ is a firmly nonexpansive, i.e., for all $x, y \in H$,

$$
\left\|T_{r}^{f} x-T_{r}^{f} y\right\|^{2} \leq\left\langle T_{r}^{f} x-T_{r}^{f} y, x-y\right\rangle ;
$$

(B3) $F\left(T_{r}^{f}\right)=E P(f)$;

(B4) $E P(f)$ is closed and convex.

Lemma $2.4([9])$. Assume that $T: C \rightarrow C$ is a nonexpansive mapping. If $T$ has a fixed point, then

(i) $F(T)$ is closed convex subset of $H$.

(ii) $I-T$ is demiclosed, i.e., whenever $\left\{x_{n}\right\}$ is a sequence in $C$ weakly converging to some $x \in C$ and the sequence $\left\{(I-T) x_{n}\right\}$ strongly converges to some $y$, it follows that $(I-T) x=y$.

\section{Main results}

In this section, we shall prove the convergence theorem for the method (3). Putting

$$
F=\left(\cap_{l=1}^{K} E P\left(f_{l}\right)\right) \bigcap\left(\cap_{i=1}^{N} F\left(S_{i}\right)\right) \bigcap\left(\cap_{k=1}^{M} V I\left(A_{k}, C\right)\right)
$$

and assume that $F$ is the nonempty set. We also propose a simpler algorithm than the algorithm (3) for a system of variational inequalities and a finite family of nonexpansive mappings. 
Theorem 3.1. Let $\left\{A_{k}\right\}_{k=1}^{M}: C \rightarrow H$ be a finite family of $\alpha$-inverse strongly monotone operators, $\left\{S_{i}\right\}_{i=1}^{N}: C \rightarrow C$ be a finite family of nonexpansive mappings, and $\left\{f_{l}\right\}_{l=1}^{K}$ be a finite family of bifunctions from $C \times C$ to $\mathbb{R}$ satisfying the conditions (A1)-(A4). Assume that the set $F$ is nonempty, $\lambda \in(0 ; 2 \alpha)$ and the control parameter sequences $\left\{\alpha_{n}\right\}$ and $\left\{r_{n}\right\}$ satisfy the following conditions:

(i) $\left\{\alpha_{n}\right\} \subset[0,1], \limsup _{n \rightarrow \infty} \alpha_{n}<1$;

(ii) $\left\{r_{n}\right\} \subset[d, \infty)$ for some $d>0$.

Then the sequence $\left\{x_{n}\right\}$ is generated by algorithm (3) converges strongly to $P_{F} x_{0}$.

Proof. We divide the proof of Theorem 3.1 into seven steps.

Step 1. We show that $F, C_{n}, Q_{n}$ are closed convex subsets of $H$. By Lemmas 2.1, 2.3, and 2.4, $E P\left(f_{l}\right), V I\left(A_{k}, C\right), F\left(S_{i}\right)$ are closed and convex. Hence, $F$ is closed and convex. From the definitions of $C_{n}, Q_{n}$, we see that $Q_{n}$ is closed and convex and $C_{n}$ is closed. Now, we show that $C_{n}$ is convex. Indeed, the inequality $\left\|v-\overline{y_{n}}\right\| \leq\left\|v-x_{n}\right\|$ is equivalent to

$$
\left\langle v, x_{n}-\overline{y_{n}}\right\rangle \leq \frac{1}{2}\left(\left\|x_{n}\right\|^{2}-\left\|\bar{y}_{n}\right\|^{2}\right)
$$

This implies that $C_{n}$ is convex for all $n \geq 0$, and so $\Pi_{C_{n} \cap Q_{n}} x_{0}$ and $\Pi_{F} x_{0}$ are well-defined.

Step 2. We show that $F \subset C_{n} \cap Q_{n}$ for all $n \geq 0$. We have $y_{n}^{i}=\alpha_{n} x_{n}-(1-$ $\left.\alpha_{n}\right) S_{i} \bar{u}_{n}$. For every $u \in F$, by the convexity of $\|\cdot\|^{2}$ and the nonexpansiveness of $S_{i_{n}}$, we obtain

$$
\begin{aligned}
\left\|u-\bar{y}_{n}\right\|^{2}= & \left\|u-\alpha_{n} \bar{u}_{n}-\left(1-\alpha_{n}\right) S_{i_{n}} \bar{u}_{n}\right\|^{2} \\
= & \|u\|^{2}-2 \alpha_{n}\left\langle u, \bar{u}_{n}\right\rangle-2\left(1-\alpha_{n}\right)\left\langle u, S_{i_{n}} \bar{u}_{n}\right\rangle \\
& +\left\|\alpha_{n} x_{n}+\left(1-\alpha_{n}\right) S_{i_{n}} \bar{u}_{n}\right\|^{2} \\
\leq & \|u\|^{2}-2 \alpha_{n}\left\langle u, \bar{u}_{n}\right\rangle-2\left(1-\alpha_{n}\right)\left\langle u, S_{i_{n}} \bar{u}_{n}\right\rangle+\alpha_{n}\left\|x_{n}\right\|^{2} \\
& +\left(1-\alpha_{n}\right)\left\|S_{i_{n}} \bar{u}_{n}\right\|^{2} \\
= & \alpha_{n}\left\|u-\bar{u}_{n}\right\|^{2}+\left(1-\alpha_{n}\right)\left\|u-S_{i_{n}} \bar{u}_{n}\right\|^{2} \\
\leq & \alpha_{n}\left\|u-\bar{u}_{n}\right\|^{2}+\left(1-\alpha_{n}\right)\left\|u-\bar{u}_{n}\right\|^{2} \\
= & \left\|u-\bar{u}_{n}\right\|^{2} .
\end{aligned}
$$

From (4), the definition of $\bar{u}_{n}$, and the nonexpansiveness of $P_{C}\left(I-\lambda A_{k_{n}}\right), T_{r_{n}}^{f_{l}}$, we have

$$
\begin{aligned}
\left\|u-\bar{u}_{n}\right\| & =\left\|P_{C}\left(I-\lambda A_{k_{n}}\right) u-P_{C}\left(I-\lambda A_{k_{n}}\right) \bar{z}_{n}\right\| \\
& \leq\left\|u-\bar{z}_{n}\right\| \\
& =\left\|T_{r_{n}}^{f_{l_{n}}} u-T_{r_{n}}^{f_{l_{n}}} x_{n}\right\| \\
& \leq\left\|u-x_{n}\right\| .
\end{aligned}
$$


From (8) and (9),

$$
\left\|u-\bar{y}_{n}\right\| \leq\left\|u-\bar{z}_{n}\right\| \leq\left\|u-x_{n}\right\| .
$$

This implies that $F \subset C_{n}$ for all $n \geq 0$. Next, we show that $F \subset C_{n} \cap Q_{n}$ for all $n \geq 0$ by the induction. Indeed, we have that $C_{0}=Q_{0}=C$ and $F \subset C=C_{0} \cap Q_{0}$. Assume that $F \subset C_{n} \cap Q_{n}$ for some $n \geq 0$. From $x_{n+1}=P_{C_{n} \cap Q_{n}} x_{0}$ and (6), we get

$$
\left\langle x_{n+1}-z, x_{0}-x_{n+1}\right\rangle \geq 0
$$

for all $z \in C_{n} \cap Q_{n}$. Since $F \subset C_{n} \cap Q_{n},\left\langle x_{n+1}-z, x_{0}-x_{n+1}\right\rangle \geq 0$ for all $z \in F$. This together with the definition of $Q_{n+1}$ implies that $F \subset Q_{n+1}$. Hence $F \subset C_{n} \cap Q_{n}$ for all $n \geq 0$.

Step 3. We show that $\left\|x_{n}-y_{n}^{i}\right\| \rightarrow 0$ and $\left\|x_{n}-z_{n}^{l}\right\| \rightarrow 0$ as $n \rightarrow \infty$ for all $i=1,2, \ldots N, l=1,2, \ldots, K$. From the definition of $Q_{n}$ and (6), we see that $x_{n}=P_{Q_{n}} x_{0}$. Therefore, for every $u \in F \subset Q_{n}$, we get

$$
\left\|x_{n}-x_{0}\right\|^{2} \leq\left\|u-x_{0}\right\|^{2}-\left\|u-x_{n}\right\|^{2} \leq\left\|u-x_{0}\right\|^{2} \text {. }
$$

This implies that the sequence $\left\{x_{n}\right\}$ is bounded. From (9), $\left\{u_{n}^{k}\right\}$ is bounded. By the nonexpansiveness of $S_{i}$, the sequence $\left\{S_{i} u_{n}^{k}\right\},\left\{y_{n}^{i}\right\}$ are also bounded.

We have $x_{n+1}=P_{C_{n} \cap Q_{n}} x_{0} \in Q_{n}, x_{n}=P_{Q_{n}} x_{0}$, from (5) we get

$$
\left\|x_{n}-x_{0}\right\|^{2} \leq\left\|x_{n+1}-x_{0}\right\|^{2}-\left\|x_{n+1}-x_{n}\right\|^{2} \leq\left\|x_{n+1}-x_{0}\right\|^{2} .
$$

Hence the sequence $\left\{\left\|x_{n}-x_{0}\right\|\right\}$ is nondecreasing, and so there exists the limit of the sequence $\left\{\left\|x_{n}-x_{0}\right\|\right\}$. From (12) we obtain

$$
\left\|x_{n+1}-x_{n}\right\|^{2} \leq\left\|x_{n+1}-x_{0}\right\|^{2}-\left\|x_{n}-x_{0}\right\|^{2} .
$$

Taking $n \rightarrow \infty$, we obtain

$$
\lim _{n \rightarrow \infty}\left\|x_{n+1}-x_{n}\right\|=0 .
$$

From $x_{n+1}=P_{C_{n} \cap Q_{n}} x_{0} \in C_{n}$ and the definition of $C_{n}$, we have that

$$
\left\|x_{n+1}-\bar{y}_{n}\right\| \leq\left\|x_{n+1}-\bar{z}_{n}\right\| \leq\left\|x_{n+1}-x_{n}\right\| \text {. }
$$

Therefore,

$$
\lim _{n \rightarrow \infty}\left\|x_{n+1}-\bar{y}_{n}\right\|=\lim _{n \rightarrow \infty}\left\|x_{n+1}-\bar{z}_{n}\right\|=0 .
$$

By (13), (14) and the estimate $\left\|x_{n}-\bar{y}_{n}\right\| \leq\left\|x_{n}-x_{n+1}\right\|+\left\|x_{n+1}-\bar{y}_{n}\right\|$, we get

$$
\lim _{n \rightarrow \infty}\left\|x_{n}-\bar{y}_{n}\right\|=0 \text {. }
$$

From the definition of $i_{n}$, we obtain

$$
\lim _{n \rightarrow \infty}\left\|x_{n}-y_{n}^{i}\right\|=0
$$

for all $i=1,2, \ldots, N$. By arguing similarly to (15), we obtain

$$
\lim _{n \rightarrow \infty}\left\|x_{n}-z_{n}^{l}\right\|=0, l=1,2, \ldots, K \text {. }
$$


Step 4. We show that $\lim _{n \rightarrow \infty}\left\|x_{n}-S_{i} x_{n}\right\|=0$. From $y_{n}^{i}=\alpha_{n} x_{n}+(1-$ $\left.\alpha_{n}\right) S_{i} \bar{u}_{n}$, we obtain

$$
\left\|x_{n}-y_{n}^{i}\right\|=\left(1-\alpha_{n}\right)\left\|x_{n}-S_{i} \bar{u}_{n}\right\| .
$$

Therefore,

$$
\begin{aligned}
\left\|x_{n}-S_{i} x_{n}\right\| & \leq\left\|x_{n}-S_{i} \bar{u}_{n}\right\|+\left\|S_{i} \bar{u}_{n}-S_{i} x_{n}\right\| \\
& \leq\left\|x_{n}-S_{i} \bar{u}_{n}\right\|+\left\|\bar{u}_{n}-x_{n}\right\| \\
& =\frac{1}{1-\alpha_{n}}\left\|x_{n}-y_{n}^{i}\right\|+\left\|\bar{u}_{n}-x_{n}\right\| .
\end{aligned}
$$

For every $u \in F$, from (4) and (6), we see that

$$
\begin{aligned}
2\left\|u-\bar{u}_{n}\right\|^{2}= & 2\left\|P_{C}\left(u-\lambda A_{k_{n}} u\right)-P_{C}\left(\bar{z}_{n}-\lambda A_{k_{n}} \bar{z}_{n}\right)\right\|^{2} \\
\leq & 2\left\langle\left(u-\lambda A_{k_{n}} u\right)-\left(\bar{z}_{n}-\lambda A_{k_{n}} \bar{z}_{n}\right), u-\bar{u}_{n}\right\rangle \\
= & \left\|\left(u-\lambda A_{k_{n}} u\right)-\left(\bar{z}_{n}-\lambda A_{k_{n}} \bar{z}_{n}\right)\right\|^{2}+\left\|u-\bar{u}_{n}\right\|^{2} \\
& -\left\|\left(u-\lambda A_{k_{n}} u\right)-\left(\bar{z}_{n}-\lambda A_{k_{n}} \bar{z}_{n}\right)-\left(u-\bar{u}_{n}\right)\right\|^{2} \\
\leq & \left\|u-\bar{z}_{n}\right\|^{2}+\left\|u-\bar{u}_{n}\right\|^{2}-\left\|\left(\bar{z}_{n}-\bar{u}_{n}\right)-\lambda\left(A_{k_{n}} \bar{z}_{n}-A_{k_{n}} u\right)\right\|^{2} \\
= & \left\|u-\bar{z}_{n}\right\|^{2}+\left\|u-\bar{u}_{n}\right\|^{2}-\left\|\bar{u}_{n}-\bar{z}_{n}\right\|^{2}-\lambda^{2}\left\|A_{k_{n}} \bar{z}_{n}-A_{k_{n}} u\right\|^{2} \\
& +2 \lambda\left\langle\bar{z}_{n}-\bar{u}_{n}, A_{k_{n}} \bar{z}_{n}-A_{k_{n}} u\right\rangle .
\end{aligned}
$$

Therefore,

$$
\begin{aligned}
\left\|u-\bar{u}_{n}\right\|^{2} & \leq\left\|u-\bar{z}_{n}\right\|^{2}-\left\|\bar{u}_{n}-\bar{z}_{n}\right\|^{2}+2 \lambda\left\langle\bar{z}_{n}-\bar{u}_{n}, A_{k_{n}} \bar{z}_{n}-A_{k_{n}} u\right\rangle \\
& \leq\left(\left\|u-\bar{z}_{n}\right\|^{2}-\left\|\bar{u}_{n}-\bar{z}_{n}\right\|^{2}\right)+2 \lambda\left\|\bar{u}_{n}-\bar{z}_{n}\right\|\left\|A_{k_{n}} \bar{z}_{n}-A_{k_{n}} u\right\| \\
& \leq\left(\left\|u-x_{n}\right\|^{2}-\left\|\bar{u}_{n}-\bar{z}_{n}\right\|^{2}\right)+2 \lambda\left\|\bar{u}_{n}-\bar{z}_{n}\right\|\left\|A_{k_{n}} \bar{z}_{n}-A_{k_{n}} u\right\| .
\end{aligned}
$$

From the convexity of $\|\cdot\|^{2}$ and the nonexpansiveness of $S_{i}$ we have

$$
\begin{aligned}
\left\|u-y_{n}^{i}\right\|^{2} & =\left\|u-\left(\alpha_{n} \bar{u}_{n}+\left(1-\alpha_{n}\right) S_{i} \bar{u}_{n}\right)\right\|^{2} \\
& \leq \alpha_{n}\left\|u-\bar{u}_{n}\right\|^{2}+\left(1-\alpha_{n}\right)\left\|u-S_{i} \bar{u}_{n}\right\|^{2} \\
& \leq \alpha_{n}\left\|u-\bar{u}_{n}\right\|^{2}+\left(1-\alpha_{n}\right)\left\|u-\bar{u}_{n}\right\|^{2} \\
& =\left\|u-\bar{u}_{n}\right\|^{2} \\
& =\left\|P_{C}\left(u-\lambda A_{k_{n}} u\right)-P_{C}\left(\bar{z}_{n}-\lambda A_{k_{n}} \bar{z}_{n}\right)\right\|^{2} \\
& \leq\left\|\left(u-\lambda A_{k_{n}} u\right)-\left(\bar{z}_{n}-\lambda A_{k_{n}} \bar{z}_{n}\right)\right\|^{2} \\
& =\left\|\lambda\left(A_{k_{n}} \bar{z}_{n}-A_{k_{n}} u\right)-\left(\bar{z}_{n}-u\right)\right\|^{2} \\
& =\lambda^{2}\left\|A_{k_{n}} \bar{z}_{n}-A_{k_{n}} u\right\|^{2}-2 \lambda\left\langle A_{k_{n}} \bar{z}_{n}-A_{k_{n}} u, \bar{z}_{n}-u\right\rangle+\left\|\bar{z}_{n}-u\right\|^{2} \\
& \leq\left\|u-x_{n}\right\|^{2}-\lambda(2 \alpha-\lambda)\left\|A_{k_{n}} \bar{z}_{n}-A_{k_{n}} u\right\|^{2} .
\end{aligned}
$$

This implies that

$$
\lambda(2 \alpha-\lambda)\left\|A_{k_{n}} \bar{z}_{n}-A_{k_{n}} u\right\|^{2} \leq\left\|u-x_{n}\right\|^{2}-\left\|u-y_{n}^{i}\right\|^{2} .
$$


We have

$$
\begin{aligned}
\left|\left\|u-x_{n}\right\|^{2}-\left\|u-y_{n}^{i}\right\|^{2}\right| & =\left|\left\|u-x_{n}\right\|-\left\|u-y_{n}^{i}\right\|\right|\left(\left\|u-x_{n}\right\|+\left\|u-y_{n}^{i}\right\|\right) \\
& \leq\left\|x_{n}-y_{n}^{i}\right\|\left(\left\|u-x_{n}\right\|+\left\|u-y_{n}^{i}\right\|\right) .
\end{aligned}
$$

By the boundedness of $\left\{x_{n}\right\},\left\{y_{n}^{i}\right\}$ and (15), we obtain

$$
\left\|u-x_{n}\right\|^{2}-\left\|u-y_{n}^{i}\right\|^{2} \rightarrow 0 .
$$

The last relation and (20) imply that

$$
\lim _{n \rightarrow \infty}\left\|A_{k_{n}} \bar{z}_{n}-A_{k_{n}} u\right\|=0 .
$$

From (18) and (19), we obtain

$$
\begin{aligned}
\left\|u-y_{n}^{i}\right\|^{2} & \leq\left\|u-\bar{u}_{n}\right\|^{2} \\
& \leq\left(\left\|u-x_{n}\right\|^{2}-\left\|\bar{u}_{n}-\bar{z}_{n}\right\|^{2}\right)+2 \lambda\left\|\bar{u}_{n}-\bar{z}_{n}\right\|\left\|A_{k_{n}} \bar{z}_{n}-A_{k_{n}} u\right\| .
\end{aligned}
$$

Therefore,

(23) $\left\|\bar{u}_{n}-\bar{z}_{n}\right\|^{2} \leq\left(\left\|u-x_{n}\right\|^{2}-\left\|u-y_{n}^{i}\right\|^{2}\right)+2 \lambda\left\|\bar{u}_{n}-x_{n}\right\|\left\|A_{k_{n}} x_{n}-A_{k_{n}} u\right\|$.

From (21), (22), (23) and $0<\lambda<2 \alpha$, we get

$$
\lim _{n \rightarrow \infty}\left\|\bar{z}_{n}-\bar{u}_{n}\right\|=0
$$

Since $\left\|x_{n}-\bar{z}_{n}\right\| \rightarrow 0$ and $\left\|x_{n}-\bar{u}_{n}\right\| \leq\left\|x_{n}-\bar{z}_{n}\right\|+\left\|\bar{z}_{n}-\bar{u}_{n}\right\|$,

$$
\lim _{n \rightarrow \infty}\left\|x_{n}-\bar{u}_{n}\right\|=0 \text {. }
$$

This together with (15), (17) implies that

$$
\lim _{n \rightarrow \infty}\left\|x_{n}-S_{i} x_{n}\right\|=0
$$

for all $i=1,2, \ldots, N$. By the boundedness of $\left\{x_{n}\right\}$, there exists a subsequence $\left\{x_{m}\right\}$ of $\left\{x_{n}\right\}$ converging weakly to $\widehat{x} \in C$. From (25) and Lemma $2.4, \widehat{x} \in$ $F\left(S_{i}\right)$ for all $i=1,2, \ldots, N$. Hence, $\widehat{x} \in \bigcap_{i=1}^{N} F\left(T_{i}\right)$.

Step 5. Now we show that $\widehat{x} \in \bigcap_{k=1}^{M} V I\left(A_{k}, C\right)$. Indeed, we have that

$$
\left\|u_{m}^{k}-\bar{z}_{m}\right\| \leq\left\|u_{m}^{k}-x_{m}\right\|+\left\|x_{m}-\bar{z}_{m}\right\| .
$$

Therefore, $\left\|u_{m}^{k}-\bar{z}_{m}\right\| \rightarrow 0$ as $m \rightarrow \infty$. Note that, we also have $u_{m}^{k} \rightarrow \widehat{x}$ and $\bar{z}_{m} \rightarrow \widehat{x}$ as $m \rightarrow \infty$. We have

$$
\begin{aligned}
\left\|\bar{z}_{m}-P_{C}\left(I-\lambda A_{k}\right) \widehat{x}\right\|^{2}= & \left\|\bar{z}_{m}-\widehat{x}\right\|^{2}+2\left\langle\bar{z}_{m}-\widehat{x}, \widehat{x}-P_{C}\left(I-\lambda A_{k}\right) \widehat{x}\right\rangle \\
& +\left\|\widehat{x}-P_{C}\left(I-\lambda A_{k}\right) \widehat{x}\right\|^{2} .
\end{aligned}
$$

Moreover, from $u_{m}^{k}=P_{C}\left(I-\lambda A_{k}\right) \bar{z}_{m}$ and the nonexpansiveness of $P_{C}\left(I-\lambda A_{k}\right)$, one has

$$
\begin{aligned}
\left\|\bar{z}_{m}-P_{C}\left(I-\lambda A_{k}\right) \widehat{x}\right\|^{2} & \leq\left(\left\|\bar{z}_{m}-u_{m}^{k}\right\|+\left\|P_{C}\left(I-\lambda A_{k}\right) \bar{z}_{m}-P_{C}\left(I-\lambda A_{k}\right) \widehat{x}\right\|\right)^{2} \\
(27) & \leq\left(\left\|\bar{z}_{m}-u_{m}^{k}\right\|+\left\|\bar{z}_{m}-\widehat{x}\right\|\right)^{2} .
\end{aligned}
$$


From (26), (27) we get

$$
\begin{aligned}
\left\|\widehat{x}-P_{C}\left(I-\lambda A_{k}\right) \widehat{x}\right\|^{2} \leq & \left\|\bar{z}_{m}-u_{m}^{k}\right\|^{2}+2\left\|\bar{z}_{m}-u_{m}^{k}\right\|\left\|\bar{z}_{m}-\widehat{x}\right\| \\
& -2\left\langle\bar{z}_{m}-\widehat{x}, \widehat{x}-P_{C}\left(I-\lambda A_{k}\right) \widehat{x}\right\rangle .
\end{aligned}
$$

Letting $m \rightarrow \infty$, we obtain

$$
\widehat{x}=P_{C}\left(I-\lambda A_{k}\right) \widehat{x} .
$$

By $(7), \widehat{x} \in V I\left(A_{k}, C\right)$ for all $k=1,2, \ldots, M$.

Step 6. We show that $\widehat{x} \in \bigcap_{l=1}^{K} E P\left(f_{l}\right)$.

Note that $\lim _{n \rightarrow \infty}\left\|z_{m}^{l}-x_{m}\right\|=0$. This together $r_{m} \geq d>0$ implies that

$$
\lim _{m \rightarrow \infty} \frac{\left\|z_{m}^{l}-x_{m}\right\|}{r_{m}}=0 .
$$

We have that $z_{m}^{l}=T_{r_{m}}^{f_{l}} x_{m}$, i.e.,

$$
f_{l}\left(z_{m}^{l}, y\right)+\frac{1}{r_{m}}\left\langle y-z_{m}^{l}, z_{m}^{l}-x_{m}\right\rangle \geq 0 \quad \forall y \in C .
$$

From (29) and (A2), we get

$$
\frac{1}{r_{m}}\left\langle y-z_{m}^{l}, z_{m}^{l}-x_{m}\right\rangle \geq-f_{l}\left(z_{m}^{l}, y\right) \geq f_{k}\left(y, z_{m}^{l}\right) \quad \forall y \in C .
$$

Taking $m \rightarrow \infty$, by (28), (30) and (A4), we obtain

$$
f_{l}(y, \widehat{x}) \leq 0, \forall y \in C .
$$

For $0<t \leq 1$ and $y \in C$, putting $y_{t}=t y+(1-t) \widehat{x}$. Since $y \in C$ and $\widehat{x} \in C$, $y_{t} \in C$. Hence, for small sufficient $t$, from (A1), (A3) and (31), we have that

$$
f_{l}\left(y_{t}, \widehat{x}\right)=f_{l}(t y+(1-t) \widehat{x}, \widehat{x}) \leq 0 .
$$

By (A1), (A4), we have that

$$
\begin{aligned}
0 & =f_{l}\left(y_{t}, y_{t}\right) \\
& =f_{l}\left(y_{t}, t y+(1-t) \widehat{x}\right) \\
& \leq t f_{l}\left(y_{t}, y\right)+(1-t) f\left(y_{t}, \widehat{x}\right) \\
& \leq t f_{l}\left(y_{t}, y\right) .
\end{aligned}
$$

Dividing both sides of the last inequality by $t>0$, we obtain $f_{l}\left(y_{t}, y\right) \geq 0$ for all $y \in C$, i.e.,

$$
f_{l}(t y+(1-t) \widehat{x}, y) \geq 0, \forall y \in C .
$$

Taking $t \rightarrow 0^{+}$, from (A3), we get $f_{l}(\widehat{x}, y) \geq 0, \forall y \in C$ and $l=1,2, \ldots, K$, i.e, $\widehat{x} \in \cap_{l=1}^{K} E P\left(f_{l}\right)$. Therefore, $\widehat{x} \in F$.

Step 7. We show that $x_{n} \rightarrow P_{F} x_{0}$. Setting $w=P_{F} x_{0}$. From (11), we get

$$
\left\|x_{m}-x_{0}\right\| \leq\left\|w-x_{0}\right\| \text {. }
$$

By the lower weak continuity of $\|\cdot\|$ we have

$$
\left\|\widehat{x}-x_{0}\right\| \leq \lim _{m \rightarrow \infty} \inf \left\|x_{m}-x_{0}\right\| \leq \lim _{m \rightarrow \infty} \sup \left\|x_{m}-x_{0}\right\| \leq\left\|w-x_{0}\right\| .
$$


By the definition of $w, \widehat{x}=w$ and $\lim _{m \rightarrow \infty}\left\|x_{m}-x_{0}\right\|=\left\|\widehat{x}-x_{0}\right\|$. This implies that

$$
\lim _{m \rightarrow \infty}\left\|x_{m}\right\|=\|\widehat{x}\| .
$$

Therefore, $\lim _{m \rightarrow \infty} x_{m}=\widehat{x}$. Assume that $\left\{x_{k}\right\}$ is an any subsequence of $\left\{x_{n}\right\}$. By arguing similarly to above proof, $x_{k} \rightarrow P_{F} x_{0}$ as $k \rightarrow \infty$. Hence, $x_{n} \rightarrow P_{F} x_{0}$ as $n \rightarrow \infty$. The proof of Theorem 3.1 is complete.

Now, we consider the ill-posed system of the operator equations

$$
A_{i}(x)=0, x \in H, i=1,2, \ldots, N,
$$

where $A_{i}: H \rightarrow H$ are possibly nonlinear operators on $H$. Let $S$ denote by the set of solutions of the system (32). An element $x^{\dagger}$ is called $x_{0}$-minimize norm solution of the system (32) if $x^{\dagger} \in S$ and satisfies

$$
\left\|x^{\dagger}-x_{0}\right\|=\min \left\{\left\|z-x_{0}\right\|: z \in S\right\} .
$$

If $x_{0}=0$, then $x^{\dagger}$ is said simply to be the minimize norm solution. Several sequential and parallel iterative regularization methods $[1,2,6,8,10]$ have been proposed for finding a solution of the system (32). Using Theorem 3.1, we also obtain the following result:

Corollary 3.2. Let $A_{i}: H \rightarrow H, i=1,2, \ldots, N$ be a finite family of $\alpha$-inverse strongly monotone mappings with the set of solutions $S$ being nonempty. The sequence $\left\{x_{n}\right\}$ is generated by the following manner:

$$
\left\{\begin{array}{l}
x_{0} \in H, \\
i_{n}:=\arg \max \left\{\left\|A_{i} x_{n}\right\|: i=1, \ldots, N\right\}, \bar{A}_{n}:=A_{i_{n}} \\
C_{n}=\left\{v \in H:\left\langle v, \bar{A}_{n} x_{n}\right\rangle \leq\left\langle x_{n}-\mu \bar{A}_{n} x_{n}, \bar{A}_{n} x_{n}\right\rangle\right\}, \\
Q_{n}=\left\{v \in H:\left\langle v, x_{0}-x_{n}\right\rangle \leq\left\langle x_{n}, x_{0}-x_{n}\right\rangle\right\}, \\
x_{n+1}=P_{C_{n} \cap Q_{n}} x_{0}, n \geq 0,
\end{array}\right.
$$

where $\mu \in(0, \alpha)$. Then $\left\{x_{n}\right\}$ converges strongly to the $x_{0}$-minimize norm solution $x^{\dagger}$ of the system (32).

Proof. Putting $C=H, \lambda=2 \mu, \alpha_{n}=0$ for all $n \geq 0, S_{i}=I, f_{l}(x, y)=0$. Using Theorem 3.1, we obtain the desired result.

Next, deals with the problem finding a common element of the set of solutions of a system of variational inequalities for $\alpha$-inverse strongly monotone operators $\left\{A_{k}\right\}_{k=1}^{M}$ and the set of fixed points of a finite family of nonexpansive mappings $\left\{S_{i}\right\}_{i=1}^{N}$. One can employ the method (3) to find this common element. We obtain the following result:

Corollary 3.3. Let $\left\{A_{k}\right\}_{k=1}^{M}: C \rightarrow H$ be a finite family of $\alpha$-inverse strongly monotone operators, $\left\{S_{i}\right\}_{i=1}^{N}: C \rightarrow C$ be a finite family of nonexpansive mappings. Assume that the set $F=\left(\cap_{i=1}^{N} F\left(S_{i}\right)\right) \cap\left(\cap_{k=1}^{M} V I\left(A_{k}, C\right)\right)$ is nonempty. 
Let $\left\{x_{n}\right\}$ be the sequence generated by the following manner:

$$
\left\{\begin{array}{l}
x_{0} \in H, C_{0}=Q_{0}=C, \\
z_{n}=P_{C} x_{n}, \\
u_{n}^{k}=P_{C}\left(z_{n}-\lambda A_{k} z_{n}\right), k=1, \ldots, M, \\
k_{n}:=\arg \max \left\{\left\|u_{n}^{k}-x_{n}\right\|: k=1, \ldots, M\right\}, \bar{u}_{n}:=u_{n}^{k_{n}}, \\
y_{n}^{i}=\alpha_{n} \bar{u}_{n}+\left(1-\alpha_{n}\right) S_{i} \bar{u}_{n}, i=1, \ldots, N, \\
i_{n}:=\arg \max \left\{\left\|y_{n}^{i}-x_{n}\right\|: i=1, \ldots, N\right\}, \bar{y}_{n}:=y_{n}^{i_{n}}, \\
C_{n}=\left\{v \in H:\left\|v-\overline{y_{n}}\right\| \leq\left\|v-z_{n}\right\| \leq\left\|v-x_{n}\right\|\right\}, \\
Q_{n}=\left\{v \in H:\left\langle x_{0}-x_{n}, x_{n}-v\right\rangle \geq 0\right\}, \\
x_{n+1}=P_{C_{n} \cap Q_{n}} x_{0}, n \geq 0,
\end{array}\right.
$$

where, $\lambda \in(0 ; 2 \alpha)$ and $\left\{\alpha_{n}\right\} \subset[0,1]$, $\limsup _{n \rightarrow \infty} \alpha_{n}<1$. Then the sequence $\left\{x_{n}\right\}$ converges strongly to $P_{F} x_{0}$.

Proof. Putting $f_{l}(x, y)=0$ for all $l=1,2, \ldots, K$ and $r_{n}=1$. Then $T_{r_{n}}^{f_{l}} x=$ $P_{C} x$ for all $x \in H$. The proof of Corollary 3.3 follows from Theorem 3.1.

However, the subset $C_{n}$ in the method (33) is complex. Moreover, the projection $P_{C_{n} \cap Q_{n}} x_{0}$ in each iterative step, in general, is difficult to find it. One assumes that $P_{C} x$ can be calculated easily $[4,13]$. To overcome the complexity caused by $C_{n}$ and $P_{C_{n} \cap Q_{n}}$, we propose the following parallel modified algorithm:

Algorithm 3.4. Let $x_{0} \in H$ be an arbitrary chosen element, $\left\{\alpha_{n}\right\}$ be in $[0,1]$, and $\lambda \in(0 ; 2 \alpha)$. Assume that $x_{n}$ is known for some $n \geq 0$.

Step 1. Calculate $z_{n}=P_{C}\left(x_{n}\right)$.

Step 2. Calculate the intermediate approximations $u_{n}^{k}$ in parallel

$$
u_{n}^{k}=P_{C}\left(z_{n}-\lambda A_{k}\left(z_{n}\right)\right), k=1,2, \ldots, M .
$$

Step 3. Find $k_{n}=\arg \max \left\{\left\|u_{n}^{k}-x_{n}\right\|: k=1, \ldots, M\right\}$. Put $\bar{u}_{n}:=u_{n}^{k_{n}}$.

Step 4. Calculate the intermediate approximations $y_{n}^{i}$ in parallel

$$
y_{n}^{i}=\alpha_{n} \bar{u}_{n}+\left(1-\alpha_{n}\right) S_{i} \bar{u}_{n}, i=1,2, \ldots, N .
$$

Step 5. Find $i_{n}=\arg \max \left\{\left\|y_{n}^{i}-x_{n}\right\|: i=1, \ldots, N\right\}$. Put $\bar{y}_{n}:=y_{n}^{i_{n}}$.

Step 6. If $\left\|\bar{y}_{n}-x_{n}\right\|=0$ then stop. Else, move to Step 7.

Step 7. Define

$$
\begin{aligned}
& C_{n}=\left\{v \in H:\left\|v-\bar{y}_{n}\right\| \leq\left\|v-x_{n}\right\|\right\}, \\
& Q_{n}=\left\{v \in H:\left\langle x_{0}-x_{n}, x_{n}-v\right\rangle \geq 0\right\} .
\end{aligned}
$$

Step 8. Perform

$$
x_{n+1}=P_{C_{n} \cap Q_{n}} x_{0} .
$$

Step 9. If $x_{n+1}=x_{n}$ then stop. Else, set $n:=n+1$ and return Step 1 . 
Clearly, in every iterative step of Algorithm 3.4, $C_{n}$ and $Q_{n}$ are either $H$ or the half spaces. Therefore, by calculating similarly in [13], we can obtain $x_{n+1}=P_{C_{n} \cap Q_{n}} x_{0}$ easily. Indeed, we see that $\left\|v-\bar{y}_{n}\right\| \leq\left\|v-x_{n}\right\|$ is equivalent to

$$
\left\langle v-\frac{x_{n}+\bar{y}_{n}}{2}, x_{n}-\bar{y}_{n}\right\rangle \leq 0 \text {. }
$$

Therefore, we obtain that [13, Algorithm 1]

$$
x_{n+1}:=P_{C_{n}} x_{0}=x_{0}-\frac{\left\langle x_{n}-\bar{y}_{n}, x_{0}-\frac{\left(x_{n}+\bar{y}_{n}\right)}{2}\right\rangle}{\left\|x_{n}-\bar{y}_{n}\right\|^{2}}\left(x_{n}-\bar{y}_{n}\right),
$$

if $P_{C_{n}} x_{0} \in Q_{n}$. Else

$$
x_{n+1}=P_{C_{n} \cap Q_{n}} x_{0}:=x_{0}+\lambda_{1}\left(x_{n}-\bar{y}_{n}\right)+\lambda_{2}\left(x_{0}-x_{n}\right),
$$

where $\lambda_{1}, \lambda_{2}$ is the solution of the system of two linear equations

$$
\left\{\begin{array}{l}
\lambda_{1}\left\|x_{n}-\bar{y}_{n}\right\|^{2}+\lambda_{2}\left\langle x_{n}-\bar{y}_{n}, x_{0}-x_{n}\right\rangle=-\left\langle x_{0}-\frac{x_{n}+\bar{y}_{n}}{2}, x_{n}-\bar{y}_{n}\right\rangle \\
\lambda_{1}\left\langle x_{n}-\bar{y}_{n}, x_{0}-x_{n}\right\rangle+\lambda_{2}\left\|x_{0}-x_{n}\right\|^{2}=-\left\|x_{0}-x_{n}\right\|^{2} .
\end{array}\right.
$$

Theorem 3.5. Let $\left\{A_{k}\right\}_{k=1}^{M}: C \rightarrow H$ be a finite family of $\alpha$-inverse strongly monotone operators and $\left\{S_{i}\right\}_{i=1}^{N}: C \rightarrow C$ be a finite family of nonexpansive mappings such that $F=\left(\cap_{i=1}^{N} F\left(S_{i}\right)\right) \cap\left(\cap_{k=1}^{M} V I\left(A_{k}, C\right)\right) \neq \emptyset$. Assume that the sequence $\left\{\alpha_{n}\right\} \subset[0,1]$ satisfies $\lim _{\sup _{n \rightarrow \infty}} \alpha_{n}<1$. Then the sequence $\left\{x_{n}\right\}$ generated by Algorithm 3.4 converges strongly to $P_{F} x_{0}$.

Proof. By arguing similarly to the proof of Theorem 3.1 we obtain $F, C_{n}, Q_{n}$ are closed convex subsets of $C$. Now, we show that $F \subset C_{n} \cap Q_{n}$. For every $u \in F$, by the convexity of $\|\cdot\|^{2}$ and the nonexpansiveness of $S_{i_{n}}$, we obtain

$$
\begin{aligned}
\left\|u-\bar{y}_{n}\right\|^{2}= & \left\|u-\alpha_{n} \bar{u}_{n}-\left(1-\alpha_{n}\right) S_{i_{n}} \bar{u}_{n}\right\|^{2} \\
= & \|u\|^{2}-2 \alpha_{n}\left\langle u, \bar{u}_{n}\right\rangle-2\left(1-\alpha_{n}\right)\left\langle u, S_{i_{n}} \bar{u}_{n}\right\rangle \\
& +\left\|\alpha_{n} \bar{u}_{n}+\left(1-\alpha_{n}\right) S_{i_{n}} \bar{u}_{n}\right\|^{2} \\
\leq & \|u\|^{2}-2 \alpha_{n}\left\langle u, \bar{u}_{n}\right\rangle-2\left(1-\alpha_{n}\right)\left\langle u, S_{i_{n}} \bar{u}_{n}\right\rangle+\alpha_{n}\left\|\bar{u}_{n}\right\|^{2} \\
& +\left(1-\alpha_{n}\right)\left\|S_{i_{n}} \bar{u}_{n}\right\|^{2} \\
= & \alpha_{n}\left\|u-\bar{u}_{n}\right\|^{2}+\left(1-\alpha_{n}\right)\left\|u-S_{i_{n}} \bar{u}_{n}\right\|^{2} \\
\leq & \alpha_{n}\left\|u-\bar{u}_{n}\right\|^{2}+\left(1-\alpha_{n}\right)\left\|u-\bar{u}_{n}\right\|^{2} \\
= & \left\|u-\bar{u}_{n}\right\|^{2}
\end{aligned}
$$

From the definition of $\bar{u}_{n},(7)$ and the nonexpansiveness of $P_{C}\left(I-\lambda A_{k_{n}}\right)$ and $P_{C}$, we have

$$
\begin{aligned}
\left\|u-\bar{u}_{n}\right\| & =\left\|P_{C}\left(I-\lambda A_{k_{n}}\right) u-P_{C}\left(I-\lambda A_{k_{n}}\right) z_{n}\right\| \\
& \leq\left\|u-z_{n}\right\|=\left\|P_{C} u-P_{C} x_{n}\right\|
\end{aligned}
$$




$$
\leq\left\|u-x_{n}\right\| .
$$

Therefore,

$$
\left\|u-\bar{y}_{n}\right\| \leq\left\|u-x_{n}\right\| .
$$

This implies that $F \subset C_{n}$ for all $n \geq 0$. By the induction, we obtain that $F \subset C_{n} \cap Q_{n}$ for all $n \geq 0$. By arguing similarly to the proof of Theorem 3.1 we obtain the sequences $\left\{x_{n}\right\},\left\{y_{n}^{i}\right\},\left\{u_{n}\right\},\left\{T_{i} u_{n}\right\}$ are bounded and

$$
\left\{\begin{array}{l}
\lim _{n \rightarrow \infty}\left\|x_{n+1}-x_{n}\right\|=0 \\
\lim _{n \rightarrow \infty}\left\|x_{n+1}-\bar{y}_{n}\right\|=0 \\
\lim _{n \rightarrow \infty}\left\|x_{n}-y_{n}^{i}\right\|=0, \forall i=1,2, \ldots, N
\end{array}\right.
$$

By $\bar{u}_{n}, T_{i} \bar{u}_{n} \in C$ and the convexity of $C, y_{n}^{i} \in C$. Hence $\left\|z_{n}-y_{n}^{i}\right\|=$ $\left\|P_{C} x_{n}-P_{C} y_{n}^{i}\right\| \leq\left\|x_{n}-y_{n}^{i}\right\| \rightarrow 0$. So, $\left\|x_{n}-z_{n}\right\| \leq\left\|x_{n}-y_{n}^{i}\right\|+\left\|y_{n}^{i}-z_{n}\right\| \rightarrow$ 0 . We have

$$
\begin{aligned}
\left\|z_{n}-y_{n}^{i}\right\| & =\left\|\alpha_{n}\left(z_{n}-\bar{u}_{n}\right)+\left(1-\alpha_{n}\right)\left(z_{n}-T_{i} \bar{u}_{n}\right)\right\| \\
& \geq\left(1-\alpha_{n}\right)\left\|z_{n}-T_{i} \bar{u}_{n}\right\|-\alpha_{n}\left\|z_{n}-\bar{u}_{n}\right\| .
\end{aligned}
$$

Therefore,

$$
\left\|z_{n}-T_{i} \bar{u}_{n}\right\| \leq \frac{1}{1-\alpha_{n}}\left\|z_{n}-y_{n}^{i}\right\|+\frac{\alpha_{n}}{1-\alpha_{n}}\left\|z_{n}-\bar{u}_{n}\right\|
$$

This together with the nonexpansiveness of $T_{i}$ implies that

$$
\begin{aligned}
\left\|z_{n}-T_{i} z_{n}\right\| & \leq\left\|z_{n}-T_{i} \bar{u}_{n}\right\|+\left\|T_{i} \bar{u}_{n}-T_{i} x_{n}\right\| \\
& \leq\left\|z_{n}-T_{i} \bar{u}_{n}\right\|+\left\|\bar{u}_{n}-x_{n}\right\| \\
& \leq \frac{1}{1-\alpha_{n}}\left\|z_{n}-y_{n}^{i}\right\|+\frac{\alpha_{n}}{1-\alpha_{n}}\left\|z_{n}-\bar{u}_{n}\right\|+\left\|\bar{u}_{n}-z_{n}\right\|+\left\|z_{n}-x_{n}\right\| \\
(37) & \leq \frac{1}{1-\alpha_{n}}\left\|z_{n}-y_{n}^{i}\right\|+\frac{1}{1-\alpha_{n}}\left\|z_{n}-\bar{u}_{n}\right\|+\left\|z_{n}-x_{n}\right\| .
\end{aligned}
$$

By arguing similarly to (24) we obtain

$$
\lim _{n \rightarrow \infty}\left\|z_{n}-\bar{u}_{n}\right\|=0
$$

From (37), (38) and $\lim _{n \rightarrow \infty}\left\|z_{n}-y_{n}^{i}\right\|=\lim _{n \rightarrow \infty}\left\|z_{n}-x_{n}\right\|=0$ we get

$$
\lim _{n \rightarrow \infty}\left\|z_{n}-T_{i} z_{n}\right\|=0 \text {. }
$$

Repeating Steps 5, 6, 7 in the proof of Theorem 3.1 we get $\lim _{n \rightarrow \infty} z_{n}=P_{F} x_{0}$. By $\lim _{n \rightarrow \infty}\left\|z_{n}-x_{n}\right\|=0, \lim _{n \rightarrow \infty} x_{n}=P_{F} x_{0}$. The proof of Theorem 3.5 is complete.

Using Theorem 3.5, one gets the following result which was obtained in [2]. 
Corollary $3.6([2])$. Let $\left\{S_{i}\right\}_{i=1}^{N}: C \rightarrow C$ be a finite family of nonexpansive mappings with $F=\bigcap_{i=1}^{N} F\left(S_{i}\right) \neq \emptyset$. Let $\left\{x_{n}\right\}$ be the sequence generated by the following algorithm:

$$
\left\{\begin{array}{l}
x_{0} \in H \\
z_{n}=P_{C}\left(x_{n}\right), \\
y_{n}^{i}=\alpha_{n} u_{n}+\left(1-\alpha_{n}\right) S_{i} u_{n}, i=1, \ldots, N, \\
i_{n}:=\arg \max \left\{\left\|y_{n}^{i}-x_{n}\right\|: i=1, \ldots, N\right\}, \bar{y}_{n}:=y_{n}^{i_{n}}, \\
C_{n}=\left\{v \in H:\left\|v-\overline{y_{n}}\right\| \leq\left\|v-x_{n}\right\|\right\} \\
Q_{n}=\left\{v \in H:\left\langle x_{0}-x_{n}, x_{n}-v\right\rangle \geq 0\right\} \\
x_{n+1}=P_{C_{n} \cap Q_{n}} x_{0}, n \geq 0,
\end{array}\right.
$$

where the sequence $\left\{\alpha_{n}\right\} \subset[0,1]$ satisfies $\lim _{\sup _{n \rightarrow \infty}} \alpha_{n}<1$. Then the sequence $\left\{x_{n}\right\}$ converges strongly to $P_{F} x_{0}$.

Proof. Putting $A(x)=0$ for all $x \in H$. The proof of Corollary 3.6 follows immediately from Theorem 3.5.

Acknowledgments. The author wishes to thank Prof. Dsc Pham Ky Anh, Department of Mathematics, Hanoi University of Science, VNU for his hints concerning this paper. The author also thanks the reviewers for their valuable comments and suggestions which improved this paper.

\section{References}

[1] P. K. Anh, Ng. Buong, and D. V. Hieu, Parallel methods for regularizing systems of equations involving accretive operators, Appl. Anal. 93 (2014), no. 10, 2136-2157.

[2] P. K. Anh and C. V. Chung, Parallel hybrid methods for a finite family of relatively nonexpansive mappings, Numer. Funct. Anal. Optim. 35 (2014), no. 6, 649-664.

[3] P. K. Anh and D. V. Hieu, Parallel and sequential hybrid methods for a finite family of asymptotically quasi $\phi$-nonexpansive mappings, J. Appl. Math. Comput. (2014), DOI:10.1007/s12190-014-0801-6.

[4] H. H. Bauschke, J. M. Borwein, and A. S. Lewis, The method of cyclic projections for closed convex sets in Hilbert space, Recent developments in optimization theory and nonlinear analysis (Jerusalem, 1995), 1-38, Contemp. Math., 204, Amer. Math. Soc., Providence, RI, 1997.

[5] E. Blum and W. Oettli, From optimization and variational inequalities to equilibrium problems, Math. Program. 63 (1994), no. 1-4, 123-145.

[6] M. Burger and B. Kaltenbacher, Regularizing Newton-Kaczmarz methods for nonlinear ill-posed problems, SIAM J. Numer. Anal. 44 (2006), no. 1, 153-182.

[7] P. L. Combettes and S. A. Hirstoaga, Equilibrium programming in Hilbert spaces, J. Nonlinear Convex Anal. 6 (2005), no. 1, 117-136.

[8] A. De Cezaro, M. Haltmeier, A. Leitao, and O. Scherzer, On steepest-descent-Kaczmarz method for regularizing systems of nonlinear ill-posed equations, Appl. Math. Comput. 202 (2008), no. 2, 596-607.

[9] K. Goebel and W. A. Kirk, Topics in Metric Fixed Point Theory, Cambridge Studies in Advanced Math., vol. 28, Cambridge University Press, Cambridge, 1990.

[10] M. Haltmeier, R. Kowar, A. Leitao, and O. Scherzer, Kaczmarz methods for regularizing nonlinear ill-posed equations, Inverse Probl. Imaging 1 (2007), no. 2, 289-298. 
[11] H. Iiduka and W. Takahashi, Strong convergence theorems for nonexpansive nonselfmappings and inverse-strongly-monotone mappings, J. Convex Anal. 11 (2004), no. 1, 69-79.

[12] S. Saeidi, Iterative methods for equilibrium problems, variational inequalities and fixed points, Bull. Iranian Math. Soc. 36 (2010), no. 1, 117-135.

[13] M. V. Solodov and B. F. Svaiter, Forcing strong convergence of proximal point iterations in a Hilbert space, Math. Program. 87 (2000), no. 1, 189-202.

[14] W. Takahashi, Weak and strong convergence theorems for families of nonexpansive mappings and their applications, Ann. Univ. Mariae Curie-Sklodowska Sect. A 51 (1997), no. 2, 277-292.

[15] _ Nonlinear Functional Analysis, Yokohama Publishers, Yokohama, 2000.

[16] S. Takahashi and W. Takahashi, Viscosity approximation methods for equilibrium problems and fixed point in Hilbert spaces, J. Math. Anal. Appl. 331 (2007), no. 1, 506-515.

[17] W. Takahashi and M. Toyoda, Weak convergence theorems for nonexpansive mappings and monotone mappings, J. Optim. Theory Appl. 118 (2003), no. 2, 417-428.

[18] X. Yu, Y. Yao, and Y. C. Liou, Strong convergence of a hybrid method for pseudomonotone variational inequalities and fixed point problem, An. St. Univ. "Ovidius" Constanta Ser. Mat. 20 (2012), no. 1, 489-504.

[19] C. Zhang, J. Li, and B. Liu, Strong convergence theorems for equilibrium problems and relatively nonexpansive mappings in Banach spaces, Comput. Math. Appl. 61 (2011), no. $2,262-276$.

Department of Mathematics

Hanoi University of Science

Hanoi, Vietnam

E-mail address: dv.hieu83@gmail.com 